\title{
Multitouch Interaction with Parallel Coordinates on Large Vertical Displays
}

JOSHUA REIBERT, German Aerospace Center (DLR), Germany and Bauhaus-Universität Weimar, Germany PATRICK RIEHMANN, Bauhaus-Universität Weimar, Germany BERND FROEHLICH, Bauhaus-Universität Weimar, Germany

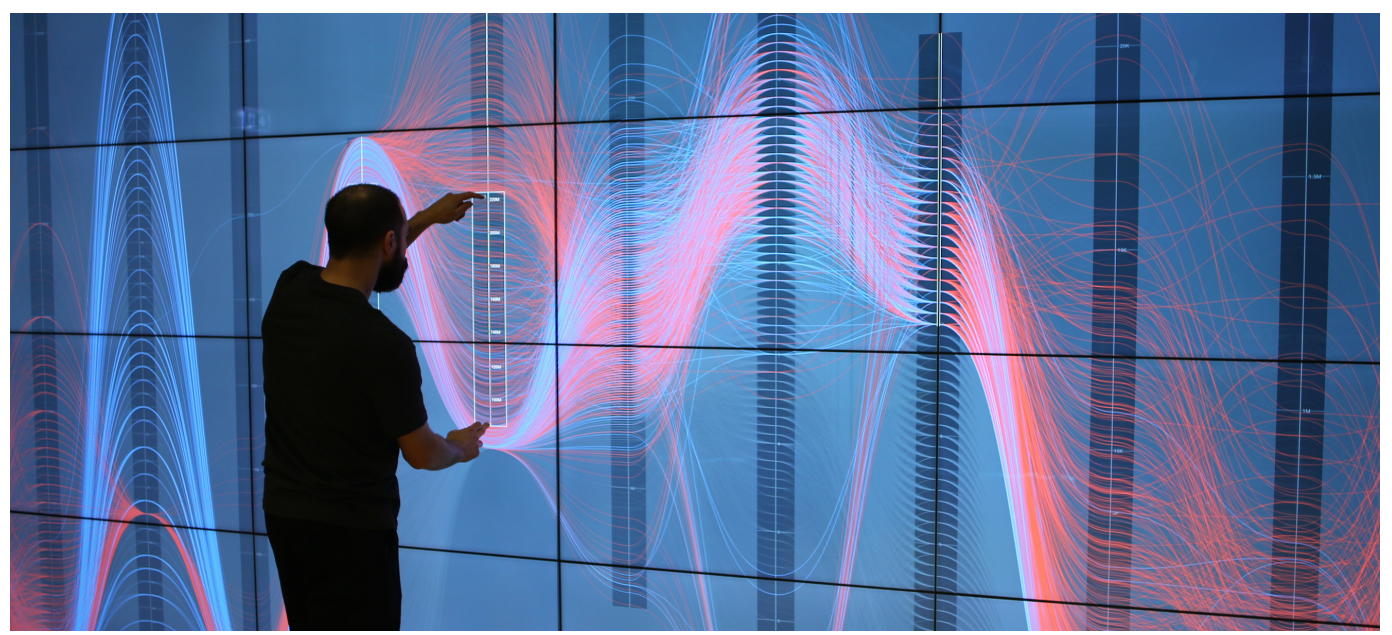

Fig. 1. Visual analysis of a movie dataset with parallel coordinates on a wall-sized display. Moving axes vertically brings regions of interest into reach. A focus area on the axis enlarges a value range to further drill down into details.

This paper presents a multitouch vocabulary for interacting with parallel coordinates plots on wall-sized displays. The gesture set relies on principles such as two-finger range definition, a functional distinction of background and foreground for applying the Hold-and-Move concept to wall-sized displays as well as flingbased interaction for triggering and controlling long-range movements. Our implementation demonstrates that out-of-reach problems and limitations regarding multitouch technology and display size can be tackled by the coherent integration of our multitouch gestures. Expert reviews indicate that our gesture vocabulary helps to solve typical analysis tasks that require interaction beyond arms' reach, and it also shows how often certain gestures were used.

Authors' addresses: Joshua Reibert, German Aerospace Center (DLR), Institute of Data Science, Mälzerstraße 3, Jena, 07745, Germany, Bauhaus-Universität Weimar, Bauhausstraße 11, Weimar, 99423, Germany, joshua.reibert@uni-weimar.de, joshua.reibert@dlr.de; Patrick Riehmann, Bauhaus-Universität Weimar, Bauhausstraße 11, Weimar, 99423, Germany, patrick. riehmann@uni-weimar.de; Bernd Froehlich, Bauhaus-Universität Weimar, Bauhausstraße 11, Weimar, 99423, Germany bernd.froehlich@uni-weimar.de.

Permission to make digital or hard copies of all or part of this work for personal or classroom use is granted without fee provided that copies are not made or distributed for profit or commercial advantage and that copies bear this notice and the full citation on the first page. Copyrights for components of this work owned by others than ACM must be honored. Abstracting with credit is permitted. To copy otherwise, or republish, to post on servers or to redistribute to lists, requires prior specific permission and/or a fee. Request permissions from permissions@acm.org.

(c) 2020 Association for Computing Machinery.

2573-0142/2020/11-ART199 \$15.00

https://doi.org/10.1145/3427327

Proc. ACM Hum.-Comput. Interact., Vol. 4, No. ISS, Article 199. Publication date: November 2020. 
CCS Concepts: • Human-centered computing $\rightarrow$ Gestural input; Visual analytics; Information visualization.

Additional Key Words and Phrases: parallel coordinates plots, focus + context, multitouch, large high-resolution displays

\section{ACM Reference Format:}

Joshua Reibert, Patrick Riehmann, and Bernd Froehlich. 2020. Multitouch Interaction with Parallel Coordinates on Large Vertical Displays. Proc. ACM Hum.-Comput. Interact. 4, ISS, Article 199 (November 2020), 22 pages. https://doi.org/10.1145/3427327

\section{INTRODUCTION}

Regular computer screens offer only a limited frame to fit information into whereas large highresolution displays (LHRDs) are human-scale environments that offer considerably more screen real-estate that information visualization can take advantage of. However, human abilities and perception impose natural limitation on the design of visualizations for these displays. Parallel coordinates especially benefit from LHRDs when visualizing a large number of attributes and items by improving legibility and reducing clutter. Even so, parallel coordinate plots (PCPs) have been used only occasionally on very LHRDs with a diagonal beyond 150 inches so far. In these cases, the PCPs were typically integrated into a coordinated multi-view framework covering only a fraction of the screen.

This motivated us to develop a multitouch gesture vocabulary for direct interaction with PCPs taking advantage of the entire screen real-estate of an LHRD. Most of our techniques build on the concept of Hold-and-Move, a bimanual interaction method with the first touch holding a frame of reference while a temporally separated second touch manipulates objects [22]. Specific enhancements for LHRDs aid in avoiding long-range swipes but also enable access beyond arm's reach. Our techniques include two-finger defined ranges for effective record selection, axes switching for adjacent and distant axis pairs using non-isomorphic control, focus + context on an axis for improved precision, lifting up and pushing down axes for comfortable access to the entire value range, and fling-based cyclic horizontal rotation as an alternative to physical walking. All these techniques were integrated into a PCP visualization system and evaluated by four experts.

We observed that using parallel coordinates on the entire LHRD resembles the typical two-step process that analysts follow when using parallel coordinates. First, an overview of the entire dataset is viewed to get a first impression of attributes and their distributions. In the next step, users tend to investigate certain attributes or attribute combinations, for instance, regarding detailed distributions or correlations. Approaching these specific axes on an LHRD creates a kind of natural focus, while all other axes, the ones that are further towards the boundary of the field of view, provide a natural context. Multitouch interaction as the most casual input modality is ideal for working directly with the data when standing in that natural focus up close to the LHRD. Of course, the size of the LHRD poses challenges in that regard such as that not each part of the visualization is easy or comfortable to reach, swipes are unpleasant for long ranges, specific gesture sets have to be learned by the users, the appropriate combination of physical and virtual navigation, and sometimes flaky touch recognition.

This paper addresses these challenges and makes the following research contributions:

- the design and implementation of a multitouch vocabulary consisting of fling-based gestures, two-finger defined ranges, and effective access methods beyond arms' reach to improve the interaction with PCPs on LHRDs

- the results of an expert review, which indicate that our vocabulary helps to overcome problems of accessibility and clutter 
The coherent integration of our gesture vocabulary into a fully functional PCP demonstrator for a wall-sized display demonstrates the potential for multitouch interaction in visual analytics interfaces and motivates the further development of such interfaces for the rich repertoire of visualization techniques.

\section{RELATED WORK}

Previous work has shown the advantages of large, high-resolution displays (LHRDs) for sensemaking [1] and tasks that are part of a visual analysis process such as comparison [34], target search [4], data exploration [35], and classification [26]. As a consequence, analysts gain more and better insights into the presented data [35]. While desktop visualizations often struggle with limited screen space, LHRDs provide more display space for larger datasets, greater data dimensionality or multiple coordinated views on the data while still offering a resolution to display details from up close. However, LHRDs shift the challenge from technological aspects to human perception [2] The distortion caused by extreme viewing angles leads to misjudgments of angles and areas [6].

The advantages of LHRDs are often attributed to physical navigation and the use of embodied resources such as spatial memory, focal and peripheral vision, and optical flow [3]. Ball et al. found that users also seem to prefer physical over virtual navigation [3, 4]. Physical navigation is especially important for users to gain on overview of the visualized data by stepping back from the display. Andrews et al. argued that users are then able to perceive the full view and take advantage of visually aggregated data representations [2]. Details that are only perceivable from up close vanish without changing the visualization itself and higher-level trends emerge. However, the advantages of physical navigation are still up for debate and user studies by Jakobsen and Hornbæk cast some doubts their extent [19]. They found neither performance improvements nor a preference of physical over virtual navigation when information spaces were too large for the display. They conclude that performance only benefits from locomotion when all information can be displayed at once. Riehmann et al. combined both virtual and physical navigation for scatterplot matrices on LHRDs which allows users to choose the method that suits their individual interaction style best [36]. We similarly provide a virtual navigation technique that complements but does not replace physical navigation to support both.

Large displays depend on novel forms of user input "beyond mouse and keyboard" [24, 38]. Multitouch input is the most natural choice for user input directly on the display. However, earlier works intended to enable multitouch interaction with visualizations have often focused on mobile devices [5, 11, 44, 45]. Sadana and Stasko designed and implemented interaction techniques to support data analysis with standard visualizations on tablet devices, most prominently for scatterplots [44]. Later, they extended their system by combining scatterplots with bar charts, line charts and parallel coordinates in multiple coordinated views [45] and compared it to Tableau's Vizable [43]. They used a combination of UI elements and gestures, since, as they argued, using too many gestures would require a bigger effort for the user to discover and remember them. Kinetica is another tablet application that employs physical metaphors to create a natural user interface to explore multivariate data with a combination of scatterplots, histograms, and pie charts [42]. Also on a tablet, Baur et al. presented TouchWave, a multitouch version of stacked graphs that improves legibility and comparing values in these graphs [5]. Drucker et al. designed a gesture-centric directtouch interface for bar charts [11]. Recently, Srinivasan et al. presented InChorus for interacting with several visualizations on a tablet device and combined touch, pen, and speech input into a multimodal visualization system [46]. All these works use multitouch input for interacting with the visualizations but the suggested techniques are primarily targeted to mobile devices and therefore focus on issues caused by lower resolutions and smaller screen sizes such as the fat-finger problem and a lack of touch accuracy. 
In 2011 Kosara presented indirect multitouch interactions with parallel coordinates using a notebook's touchpad for brushing including angular brushing and brushing multiple axes simultaneously as well as inverting and reordering axes [21]. Two fingers on an axis define a range for brushing which can also be performed simultaneously on a second axis. One finger on a single axis and two more on an adjacent axis define an angular brush whereas three fingers on a single axis drag it for reordering. Later that year, Yu and Yuan presented a touch-based implementation of parallel coordinates on the first iPad [49]. They implemented a less complete interaction set to directly drag axes with a single finger, a vertical pinch between axes to reduce or increase the space between the axes, and lasso-brushing with a single finger between axes. Our pinch-based brushing is similar to the one shown by Kosara but our gesture set for LHRDs is designed to extend brushing and axis dragging beyond arm's length.

An early example focusing on a larger single screen was ScatterTouch [16] in which a single scatterplot is shown on a tabletop display and users can apply focus + context [13] with fisheye distortion to create multiple focus regions in a single plot. A similar concept (also with scatterplots) was presented by Büring et al. [8]. SketchSliders by Tsandilas et al. enables users to sketch range sliders on a tablet to interact with visualizations on an LHRD [47]. They include branched sliders to increase the granularity of subranges on a slider and transformation sliders to define a fisheye transformation for focus + context. Recently, Chegini et al. experimented with scatterplots and scatterplot matrices on a large vertical display [9], porting ScatterDice of Elmqvist et al. to a touch interface [12]. Applying the concept of overview and detail, the matrix is used as the overview while any scatterplot inside the matrix can be selected as a detail view to interact with. Riehmann et al applied multitouch gestures to time series visualizations for enabling focus + context to enlarge time ranges as well as individual series by increasing their vertical space and dynamically adapting them [37]. In our implementation, focus + context is also used to reduce clutter on axes and to drill down into details.

LHRDs come with new challenges such as the reachability of the upper and lower parts of a wall-sized display and the perceptual issues of distant screen content. The WallPad of Gilliot et al. addresses reachability and provides a virtual pointer for accessing remote content [14]. Lischke et al. proposed a technique called Windows Spinning that allows users to move the whole screen from left to right with mouse and keyboard [25] which was inspired by the Tablecloth of Robertson et al. [40]. Riehmann et al. proposed the paternoster technique for a scatterplot matrix that additionally allows to move the screen vertically to bring the upper and lower areas into reach [36]. We offer a similar scrolling technique in the horizontal direction but our gesture set also allows dragging of axes in vertical direction as well as extending the vertical reach during interaction on an axis.

In order to provide remote interaction with visualizations on LHRDs, many recent publications have focused on remote interaction with mobile devices such as smartphones and tablets. SleeD was designed by von Zadow et al. and is a sleeve multitouch display attached to the non-dominant arm as a private detail view [48]. However, users reported it is hard to use for very high or low wall positions and requires switching between displays. Dachselt and Buchholz minimize such gaze shifts by using the built-in accelerometer of mobile devices to detect tilt gestures for zooming and panning [10]. Kister et al. showed standard graph visualizations on a wall display with space-aware mobile devices as personal views for interaction [20]. The large display gives an overview and the mobile devices offer detailed views. Prouzeau et al. investigated selection techniques for graph exploration with user pairs and proposed a technique to propagate selections in the graph to help them solve a task together [32]. Langner et al. focused on different visualizations in coordinated views with mobile devices and direct touch gestures to support visual exploration [23]. Since both make use of touch input, they developed a uniform interaction vocabulary with a small set of gestures for direct and remote interaction. Horak et al. used smartwatches to support remote visual

Proc. ACM Hum.-Comput. Interact., Vol. 4, No. ISS, Article 199. Publication date: November 2020. 
analysis on the wall in a similar way [18]. All these approaches enable interaction from varying distances and therefore promote physical navigation but come at the cost of relying on additional hardware such as tracking systems or depth cameras. In contrast, we focus on direct touch input as the first and foremost way to directly interact with visualizations on such screens which can eventually be complemented by remote interaction.

Mid-air gestures enable users to interact hands-free with LHRDs from a distance. Nancel et al. analyzed and tested interaction techniques for mid-air panning and zooming and recommend bimanual interaction, linear instead of circular gestures, and a high level of guidance [29]. Yet, they also observed that mid-air interaction is less efficient and more prone to fatigue than devicebased techniques. Since vision-based recognition of mid-air gestures is susceptible to lighting and occlusion, Haque et al. used a consumer device strapped to the forearm that tracks motion and orientation with an inertial measurement unit (IMU) and detects hand poses through muscle activation using Electromyography (EMG) for pointing and clicking on a large display [15]. Although their technique introduced a delay in comparison to motion tracking, they conclude that such a device is practical for distant interaction. Liu et al. presented Gunslinger which tracks hand postures of the relaxed arms with two Leap Motion devices mounted on each thigh to enable remote interaction [27]. Their interaction vocabulary allows users to trigger commands with specific hand gestures as well as rate-based control for continuous interaction such as pointing and zooming based on the distance between fingers. In contrast to other approaches, the subtle hand gestures are less tiring and require less physical space which allows them to be used simultaneously with direct touch input on an LHRD.

\section{VISUAL AND INTERACTION DESIGN}

Parallel coordinates plots (PCPs) are a common and popular visualization technique for multivariate data and typically used very early in data exploration [17]. Traditional display sizes limit the number of attributes as well as the number of data items that can be visualized. Too many attributes reduce the space between axes and therefore make it increasingly difficult to analyze the relations between attributes. On the other hand, too many data items result in overplotting as polylines clutter and thereby occlude patterns in the data. With a much larger display space as well as a higher resolution, LHRDs can mitigate both of these issues. However, interacting with such a large display introduces new challenges as it exceeds a user's physical range.

\subsection{Interacting with Parallel Coordinates}

Interaction is especially important for PCPs since many parameters of the visualization must be manipulated in order to answer specific questions about or detect patterns within the dataset. Brushing is the most common interaction with PCPs to select a subset of items which can then be used for subsequent operations such as highlighting, displaying labels, and filtering [17]. In the simplest case, brushes are axis-aligned and therefore select all items in a specific value range of an attribute. Brushing on an axis requires high precision as it corresponds directly to values in the data domain. Additionally, individual items can be selected directly (probing), as well as by brushing on the area between axes to select items whose polylines intersect a rectangle (area brush) or line (lasso brush), and items within a certain slope (angular brush) [33]. Higher-dimensional brushes combine multiple brushes with logical operations to allow more complex selections. Sketch-based brushing allows users to sequentially click on multiple axes to create an n-dimensional brush with initially fixed intervals on each axis that can be interactively adjusted later on [39].

Interaction with axes in PCPs can be categorized in translation and scaling [17]. The order of axes is usually chosen arbitrarily when constructing the PCP but determines the pairwise relations that are visualized. As a result, users have to be able to reorder axes which is commonly performed 
with a drag-and-drop interaction. The horizontal positions of axes are often equidistant to put equal emphasis on all attributes but can also be chosen freely. In contrast to brushing, less precise control over the translation is required since it only determines the position of an axis on screen By default, the minimum value of an attribute intersects the corresponding axis at the bottom and the maximum at the top which allows to see patterns across attributes of different domains and units. However, when adjacent axes share the same unit, vertical translation can align them to a common value. Similarly, scaling can align them to a common scale and also reduce overplotting in dense areas. Flipping an axis is a special case of scaling that inverts the values and therefore also the patterns of negative and positive correlations.

\subsection{Design Goals}

We believe that parallel coordinates visualizations can benefit from the increased display size and resolution of LHRDs since they can accommodate more attributes and data items. Nevertheless, interaction with these displays also introduces new challenges that have to be considered carefully In our work we focus especially on the two issues of accessibility and the avoidance of prolonged touch input which we consider the most important obstacles for using multitouch input on LHRDs and derive the following design goals:

Extend reachability. Wall-sized displays exceed the reach of users and some parts of the screen might be completely out of reach. Our display is $2.31 \mathrm{~m}$ high and the lower quarter is only accessible by uncomfortable crouching while the upper part cannot be reached at all by most users. When the PCP spans the full height of the display, users cannot access data items towards the upper areas of axes, for instance when brushing on an axis. In contrast, users can always access all display areas horizontally. Yet, users may have to walk back and forth a lot when they are switching focus between axes. When they are close to the display, very distant axes may not even be visible since acute viewing angles affect perception.

Avoid long-range movements. On mobile devices, dragging an object using touch input across the whole display can already be uncomfortable and error-prone. Large display walls amplify these effects, for instance, when trying to drag an object to an intended target position on the glass surface across several meters. Desktop-based PCPs typically use drag and drop to reorder axes which does not translate well to LHRDs. Any horizontal touch interaction beyond arm's length is only possible while walking. However, that increases the risk of accidentally lifting the touch. Additionally, LHRDs usually have a blunt glass surface in front of the screen that provides the touch functionality and protects the underlying display which makes prolonged touch input very unpleasant to users. As a consequence, long-range movements should be avoided on LHRDs and substituted by alternatives.

We have established strict design rules to ensure consistency between the individual techniques and to integrate them smoothly. There are three principles that we rely on: (1) Two fingers define a range. During prototyping it was observed that users expect to define a range with a bimanual pinch gesture which was also used in related works [21, 49]. (2) Long-range touch input should be substituted with fling controls or non-linear mappings. Dragging over large distances was observed to be error-prone and uncomfortable. The fling gesture is a short-range alternative to directed long-range gestures when exact precision is not required and was already applied for navigating a scatterplot matrix on an LHRD [36]. Non-linear mappings allow continous shortrange gestures to have precise control at first but trade precision for a larger effect as the distance increases. (3) Sequential touch events of the left and the right hand are used for two-handed

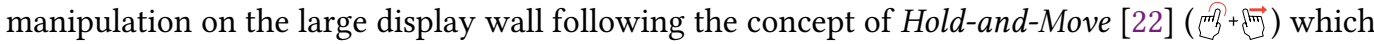


was originally proposed for hand-held devices to avoid disruptive long taps. The first touch eventusually performed with the non-dominant hand-fixates the background and provides a reference frame for subsequent touch events. Users can then manipulate foreground objects with their dominant hand and move them directly without requiring any dwell time on the object first. Due to this temporal sequence, Hold-and-Move can easily be detected and distinguished from simultaneous multitouch inputs.

\subsection{A multitouch vocabulary for LHRDs}

We developed a multitouch vocabulary in an iterative design process based on rapid prototyping. Our interactions are designed to overcome accessibility problems and limitations associated with multitouch technology and display size. The interaction techniques are designed to enable users to (1) navigate virtually to complement physical navigation and manipulate the horizontal axes' positions even over large distances, (2) shift axes vertically at any position to bring regions of interest into a comfortable interaction range, (3) define axis-aligned brushes and create focus areas on axes to analyze a specific value range in detail and reduce clutter, (4) as well as to extend those ranges intuitively beyond reach for full vertical access.

In our vocabulary, we make use of the concept of Hold-and-Move to move axes relative to the background or the other way around. Furthermore, we extended the concept with Hold-and-Fling

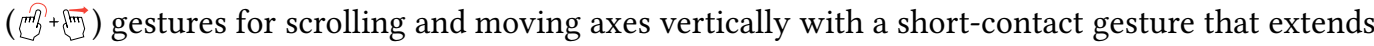
range. The overview of the gesture vocabulary can be seen in Figure 2 and the supplementary video. The particular gestures were designed based on our design goals and the principles for the gestures in subsection 3.2 and are described in detail below.

Accessing Axes Vertically. The PCP stretches the full size of the display initially and therefore enables even distant observers to see patterns in the data. But for touch interaction from up close, the lower and upper parts of the axes are out of reach. Therefore, we allow analysts to fixate the background with a hold between the axes and then to drag an axis easily up or to down (see Figure 2A and Figure 3) which is based on the Hold-and-Move concept. While this will partially move the axis off-screen, it brings a region of interest at full resolution into a convenient vertical position for interaction. When users want to bring an axis into reach even quicker, they can hold the background as before but perform a vertical fling. As a discrete alternative, a downwards fling will move the axis down to a predefined reachable position that is aligned with the tiled display grid (see Figure 2B). Similarly, an upwards fling translates the axis to an upper position such that the lower region is easily accessible. Resetting an axis to its original position is enabled with the fling gesture as well; an axis that was moved down before can be reset by flinging upwards and vice versa.

Navigating the Plot. Physical navigation allows analysts to focus on any axis by walking to it. However, frequently switching focus between attribute axes can be cumbersome and require the analyst to walk back and forth a lot. As an alternative, we propose a cyclic horizontal scrolling with a horizontal fling gesture to bring axes of interest to the user (see Figure 2C) which is based on our principle to replace long-range interaction with short-range fling gestures. By varying the strength of the fling gesture, the amount of scrolling can be controlled. The transition of the axes to their new position is animated to allow users to comprehend the position shift. In contrast to traditional parallel coordinates, our plot is cyclic and the rightmost and leftmost axis are connected by curves as well. As a result, cyclic scrolling does not alter the representation itself but creates a smooth and seamless transition. 

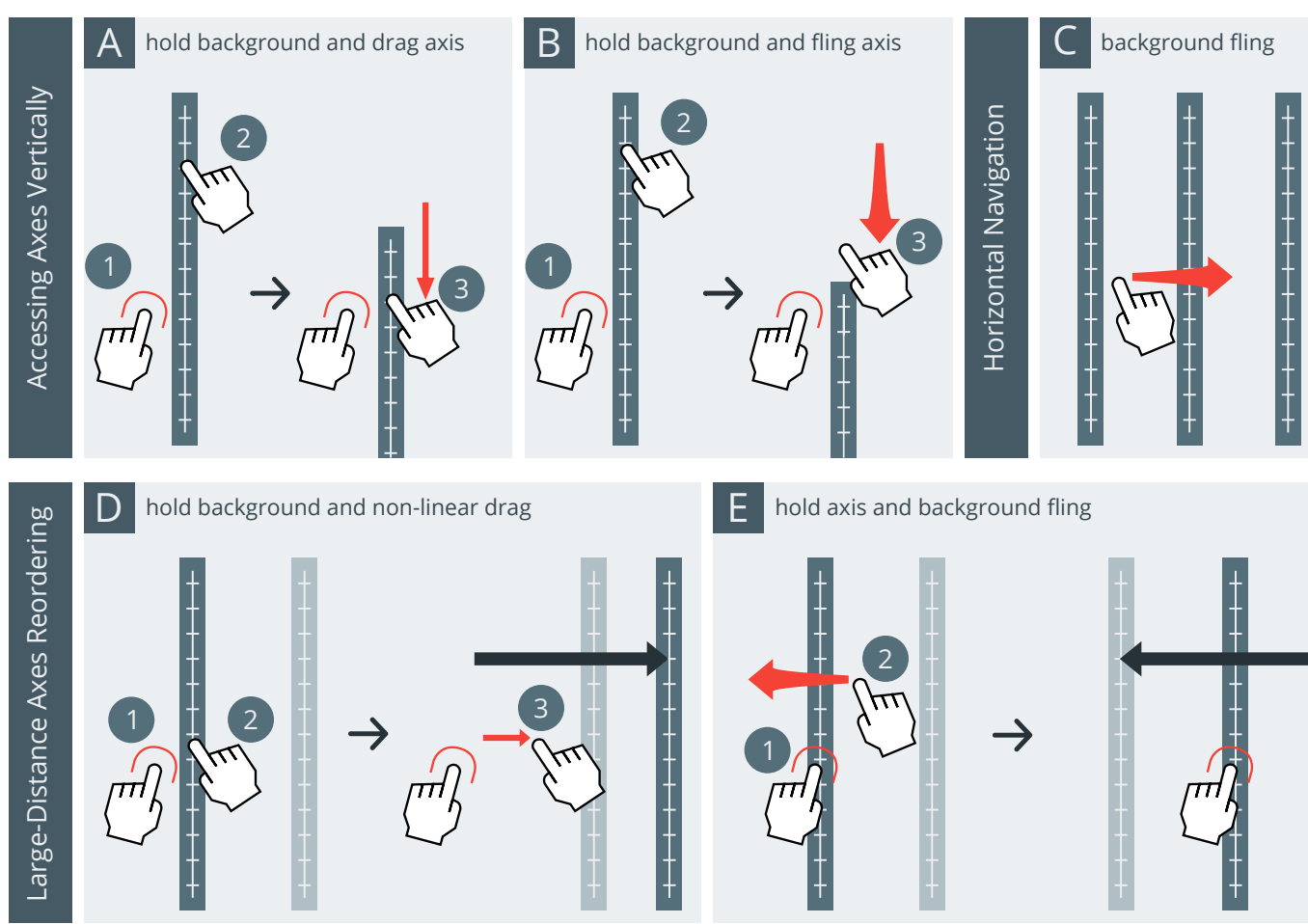

D hold background and non-linear drag

E hold axis and background fling
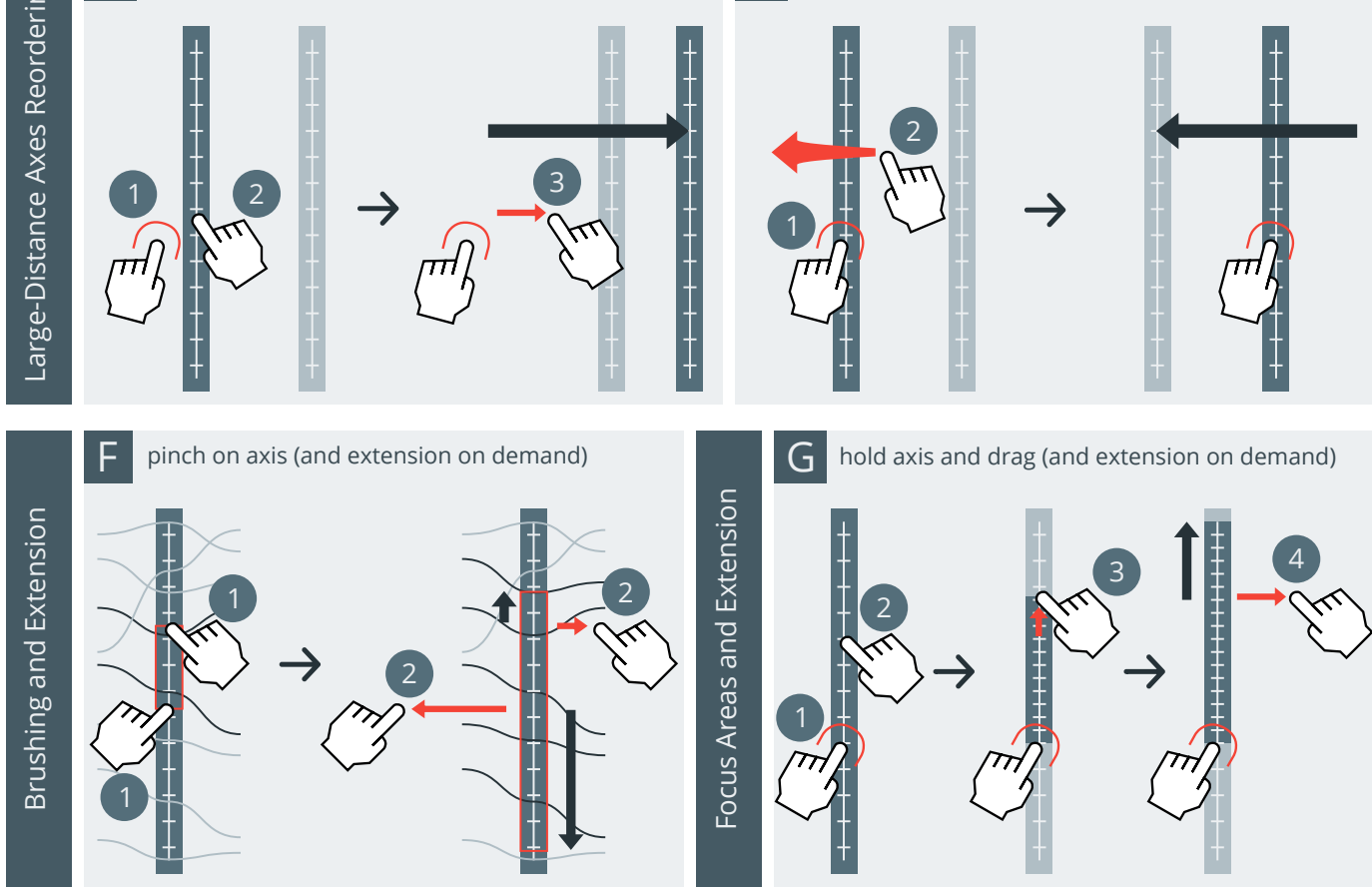

Fig. 2. Overview of the proposed touch gestures for handling parallel axes on a wall display and how they are triggered. Fling gestures $(\longrightarrow)$ as in B, C, and E replace long-range movements. Direct movements $(\longrightarrow)$ are for local adjustments as in A, F, and G. Both are usually started with a prior hold gesture $(\cap)$ to set the stationary element which could be either the background or an axis. Navigation is an exception to this rule since background and foreground are moved all along. Indirect movements $(\rightarrow)$ of an axis or a range are triggered by a local fling $(E)$ or non-linear drag gesture (D, F, and $G$ ). Defining a range always uses two fingers either for brushing $(\mathrm{F})$ or defining a focus area $(\mathrm{G})$. The range gestures differ in how they are approached; both fingers simultaneously (brushing) as in $\mathrm{F}$ or one finger holding first and then other one when creating a focus area in $\mathrm{G}$. In both cases ( $\mathrm{F}$ and $\mathrm{G}$ ) the range can be further extended beyond reach with the same extension technique. The idea is to turn the sliding direction when reaching an uncomfortable position from upwards to sideways (or downwards to sideways) and to continue sliding the finger until the upper (or lower) border of the range is in the destined position. 


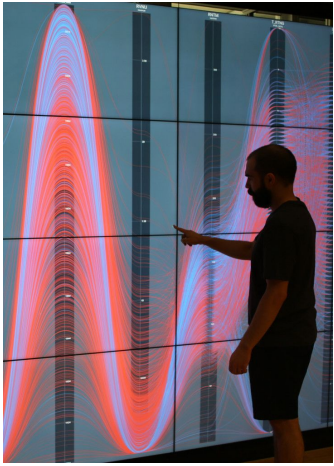

(a)

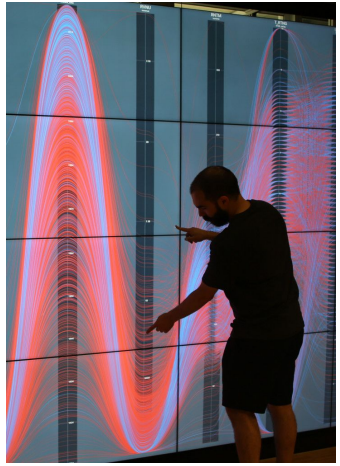

(b)

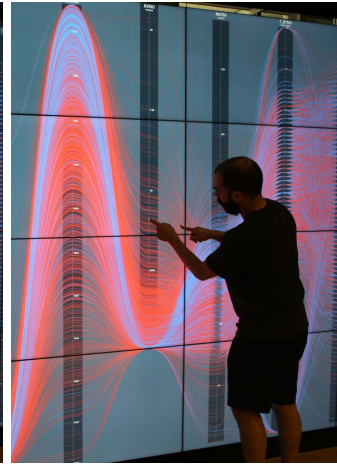

(c)

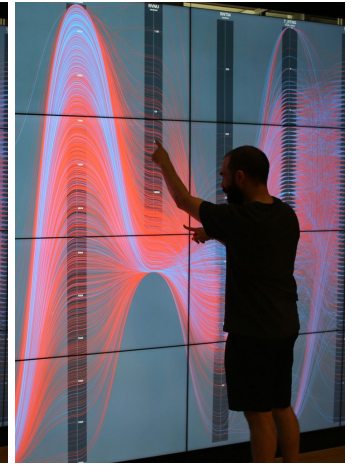

(d)

Fig. 3. The height of the display exceeds the typical users' vertical reach for touch interaction. Users can fixate the background with one finger (a) and start a drag gesture on the axis with the other hand (b). The drag moves the axis up (c) and brings the low values into reach (d).

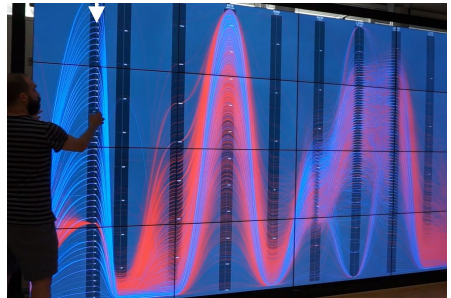

(a)

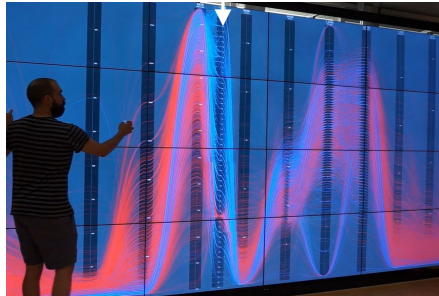

(b)

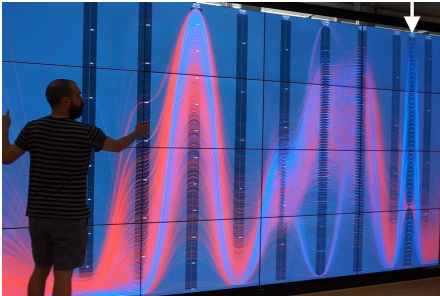

(c)

Fig. 4. Users can reorder axes over extended distances. The white arrow was added to the pictures to show the current position of the moved axis. The background is held with one finger and a subsequent drag moves the axis directly at first (a). After a distance threshold, the drag's movement uses a hyperbolic mapping to increase reach (b). This allows users to drag an axis even across the whole screen without physically moving (c).

Large-Distance Axes Reordering. The order of axes in a PCPs affects the patterns and relations between attributes that are visualized. While the initial axes layout is often arbitrarily chosen, users usually want to inspect certain pairwise relations [17]. Parallel coordinates on smaller displays without any reachability issues often make use of drag and drop gestures for reordering axes. However, on large screens, this exceeds the users' range since it would require long drags-possibly across the whole width of the display.

In our system, a single axis can be moved to a specific horizontal position based on Hold-and-Move by holding the plot background with one hand and dragging the axis with the other (see Figure 2D and Figure 4). To avoid long-range dragging, the axis moves directly with the finger at first but beyond a threshold of $15 \mathrm{~cm}$ it is amplified by the hyperbolic function $f(x)=t+\sinh ((x-t) \mathrm{s}) / \mathrm{s}$ where $t=.15$ is the threshold and $s=5$ is a scaling factor that determines the strength of the hyperbolic mapping (see Figure 5). Whenever the dragged axis crosses another axis, the polylines update automatically to reflect the changed order. While the hyperbolic mapping reduces precision for larger distances, it allows users to drag an axis over the full width of the screen without changing 


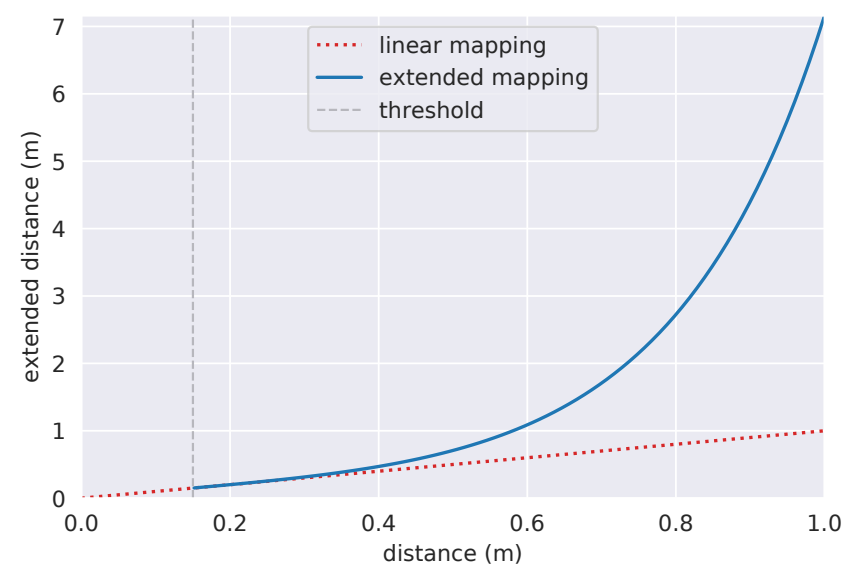

Fig. 5. The transfer function that extends the distance between the initial position of the axis and the drag gesture. Below a threshold of $15 \mathrm{~cm}$ it is linear but the hyperbolic mapping beyond that threshold allows users to move an axis over multiple meters.

their location. Precise placement is not required since the interaction only affects the order of axes and an equidistant axes layout is automatically restored once the drag gesture ends. The threshold and the scaling factor were determined to have an almost linear mapping for about the first $30 \mathrm{~cm}$ and then extend the distance over the full width of the screen when the user extends their arm. Hence, the scaling parameter is display-dependent and needs to be adapted to the respective width of a specific screen setup.

Although this allows moving a single axis to a distant location, users still have to walk to this very location afterwards in order to proceed with their analysis. Hold-and-Move gives us the opportunity to speed up the process by combining reordering and navigation. Users can hold an axis with the non-dominant hand and perform the cyclic scrolling described above (multiple times, if necessary) to move all axes along except the one that is being held and release it once another axis of interest is next to it (see Figure 2E and Figure 6). Both of these variants can be combined in any order to achieve all desired layouts of the axes.

Focus Areas on Axes. Overplotting is one of the most common issues of traditional parallel coordinates [17]. Clusters of items with similar values can hide patterns in the data even with high-resolution displays. In an effort to tackle this issue, we propose a focus + context technique to increase the space for regions of interest while compressing the upper and lower context regions. Holding an axis with one finger in combination with a subsequent drag gesture defines the initial value range and ties the focus area to both fingers in accordance to our principle that two fingers define a range. Moving both fingers apart, the focus region is stretched and the context regions to either side are compressed to the remaining space (see Figure $2 \mathrm{G}$ and Figure 7 ). The focus region is highlighted as such with an outline and the tick labels will adapt to the new granularity. When lifting the fingers, the focus region stays in place and offers a more detailed view into the data Focus regions can be iteratively created to drill down even more into details and reveal previously hidden patterns and details. A double tap on a focus area removes it and restores the original axis. 


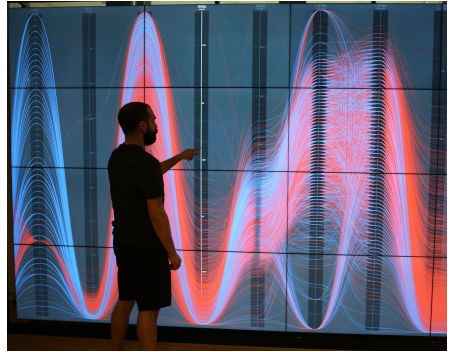

(a)

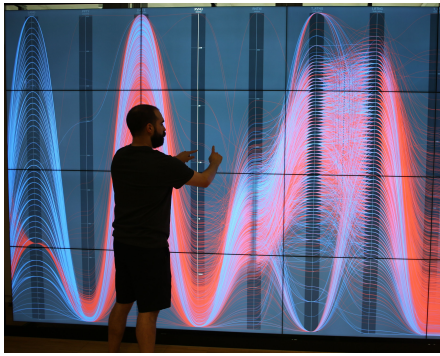

(b)

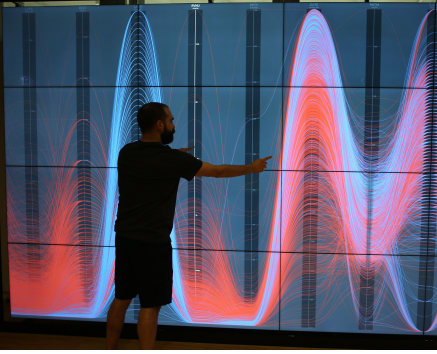

(c)

Fig. 6. When an axis is held with one hand (a), the user can still perform a horizontal fling gesture with the other hand (b) which will scroll the entire plot with all axes except the one that is being held and thereby reorders the axes (c). Applying the fling without holding an axis results in a cyclic navigation without reordering.

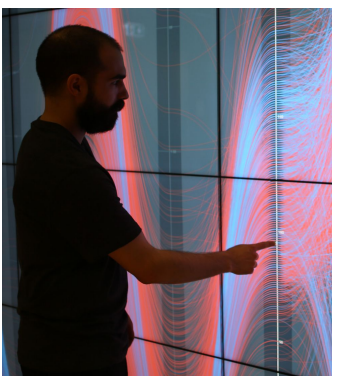

(a)

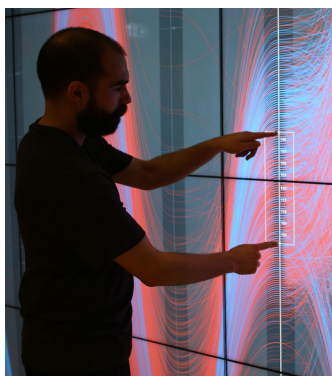

(b)

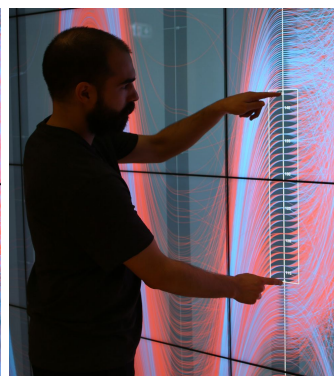

(c)

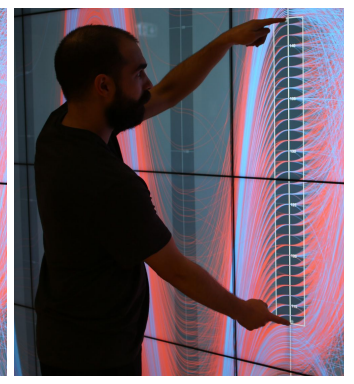

(d)

Fig. 7. Focus + context allows users to drill into details and tackle overplotting. A hold gesture with one hand fixates an axis (a). A subsequent touch with the other hand creates a focus area between the two fingers (b) and the focus area resizes accordingly with the fingers (c). Moving the fingers apart stretches the focus area and compresses the context regions below and above it $(d)$.

Data Selection, Filtering and Details. Users can select subsets of data items by defining an axisaligned brush with two fingers based on our principle for ranges. When brushes on multiple axes are active, the intersection of individual selections determines the global selection of items. More advanced brushes and other logical operations for higher-dimensional brushes are currently not supported. While selected items are drawn as usual, unselected items are drawn in the background in a light gray and with reduced opacity. A single tap inside a selected item range on an axis shows a popup with detail information for the items. For less than 20 selected items, the exact values for the attribute on that axis are displayed. When more items are selected, statistics are displayed that adapt to the attribute type of the axis. For quantitative attributes descriptive statistics such as min, mean, max, standard deviation and the quantiles are shown.

Vertical range extension for range definitions. Both the creation of focus areas as well as brushing rely on two-handed gestures for defining a value range on a vertical axis. Since the axes' vertical extent exceeds a user's reach, a desired selection is sometimes not possible directly on the axis. Alternatively, one could shift the whole axis vertically first and then do the selection. However, 


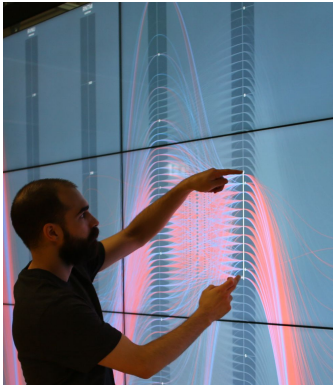

(a)

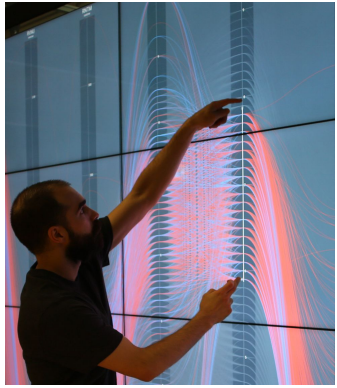

(b)

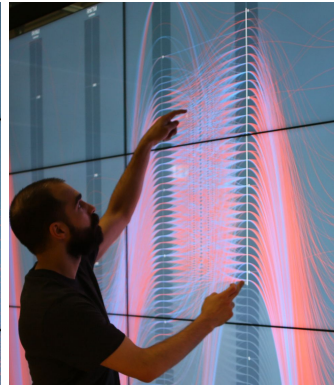

(c)

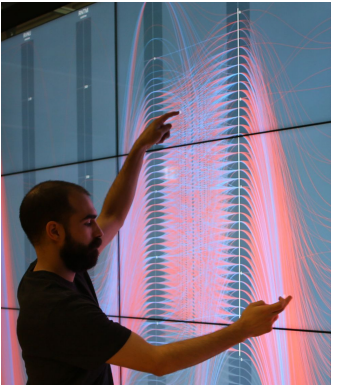

(d)

Fig. 8. A bimanual pinch gesture brushes items in the given value range (a) but users are limited by their reach which may only become evident during the interaction (b). By moving the finger away from the axis, the horizontal distance is non-linearly mapped to increase the defined range (c). This vertical range extension allows users to extend brushes in both directions $(\mathrm{d})$.

that requires two discrete steps and therefore planning ahead and does also not allow long-range brushes reaching from top to bottom.

For avoiding interruptions during brushing and to allow such long-range brushes, we propose a natural extension (see Figure 2F,G and Figure 8) that increases the range beyond reach without lifting the fingers from the screen simply by turning the sliding direction from upwards or downwards to sideways until the upper (or lower) border of the range is in the destined position. The horizontal distance of the touch points extends the vertically selected range using the same hyperbolic transfer function as the axes reordering (see Figure 5). While a selection directly on the axis is mapped linearly and therefore has a high precision, the further a touch point moves away from the axis the more it extends the current range at the cost of reducing precision.

\subsection{Visual Appearance}

The design of visualizations on LHRDs should regard the unique properties of such a display. Our PCP uses a dark color palette since bright colors can lead to eye strain during longer analysis. This is especially true with IR-based touch systems when direct sunlight is shut out and the work environment is therefore darker. While traditional parallel coordinates usually use thin lines as axes, these are harder to spot and to interact with on LHRDs. Our system uses wider axes that come with a certain physicality and are easier to touch, grab and drag. Moreover, brushes can be spanned easily and focus areas are easier to spot.

In that regard many parameters of the visualization such as the axes' width, the amount of ticks and also the touch gesture recognition use meters as a unit in order to support displays of different sizes. Knowing the measurements of the display, these are converted back to pixels for drawing. For example, the axes in our application are $10 \mathrm{~cm}$ wide as the width also indicates the range in which touch gestures are tied to the axis.

\subsection{Handling Touch Input}

Recent multitouch devices use USB-HID for communicating their touch events. Our application reads these events using the generic input event interface (evdev) provided by the Linux kernel. A unique ID identifies individual touch points and is used to store position information as well as velocity or even pressure when available. Additionally, timestamps of the initial touch down 
and the touch up events when the finger was lifted are recorded. Gesture recognition builds on this information to detect higher-level gestures of single touch points like taps and drags or even multiple touch points like pinches. At first, a touch point or group of touch points cannot be certainly identified as a gesture. Gesture recognizers watch all new touch points for specific patterns that are typical of the respective gesture. For example, the touch point of a tap must not travel more than a specified distance and exceed a certain amount of time it is active. Once a gesture is recognized, the gesture recognizer creates a gesture and passes it to the application. The visualization view can accept the gesture and any other potential gestures with that touch point are blocked. To lower the obstacle of learning new gestures, we focus on well-known gestures from mobile devices. Currently, recognizers for taps, double taps, holds, drags, pinches and flings exist.

\section{EXPERT REVIEW}

The COVID-19 pandemic and the contact restrictions did not allow us to perform a formal user study for our multitouch gesture vocabulary for PCPs. Instead, we invited four experts to review and evaluate our system and to test for and identify potential usability issues. We followed the official regulations as published by our university and ensured that only one experimenter and one participant were present at a time to minimize contacts. For the evaluation, we had three central research questions:

(1) Usability of the interface: Are experts able to perform and learn the touch gestures quickly?

(2) Comprehensibility of the visualization: Are our enhancements of the parallel coordinates plot concept concise and seamless?

(3) Effectiveness of the system: Are users able to solve typical data analysis tasks?

\subsection{Hardware Setup}

We performed the study on a tiled-display wall ( $4.08 \mathrm{~m}$ wide and $2.31 \mathrm{~m}$ high) with a total resolution of $7680 \times 4320$ pixels. It consists of 16 individual displays arranged in a $4 \times 4$ matrix. A custom-built PQLabs G4 touch overlay detects touch input on a glass surface in front of the display using IR emitter/receiver pairs. The PQLabs Linux driver [31] provides touch information using USB-HID. The display is powered by a single machine with four NVIDIA Quadro M6000-each providing a $4 \mathrm{~K}$ image for one quadrant of the whole display.

\subsection{Experimental Design}

We conducted expert reviews with four participants ( 3 male, 1 female) who work as academic researchers and have a degree in computer science. $\mathrm{P} 1$ is a senior researcher and has more than 10 years of experience in $\mathrm{HCI}$ and gestural interaction. $\mathrm{P} 2$ and $\mathrm{P} 4$ are $\mathrm{PhD}$ candidates in the field of information visualization and P3 is a research assistant. All three have at least three years of research and work experience in the field. The participants had not used our system before but were familiar with parallel coordinates and thus were able to evaluate and judge the suitability of our proposed gestures, techniques and enhancements to the PCP.

We created a dataset that combines movie data from the two online movie databases $I M D b$ and $T M D b$ as of May 2020. The dataset contains 7122 movies with the ten attributes budget, original language, popularity, release date, revenue, runtime as well as the average rating and the number of ratings on each IMDb and TMDb.

The whole expert review took around one hour per participant. Initially, the system was introduced with an explanation of the PCP and the visualized dataset and a demonstration of the gesture set. This was followed by a free exploration phase in which the participants applied all available interactions and were encouraged to ask questions if necessary. After about 15 minutes, 


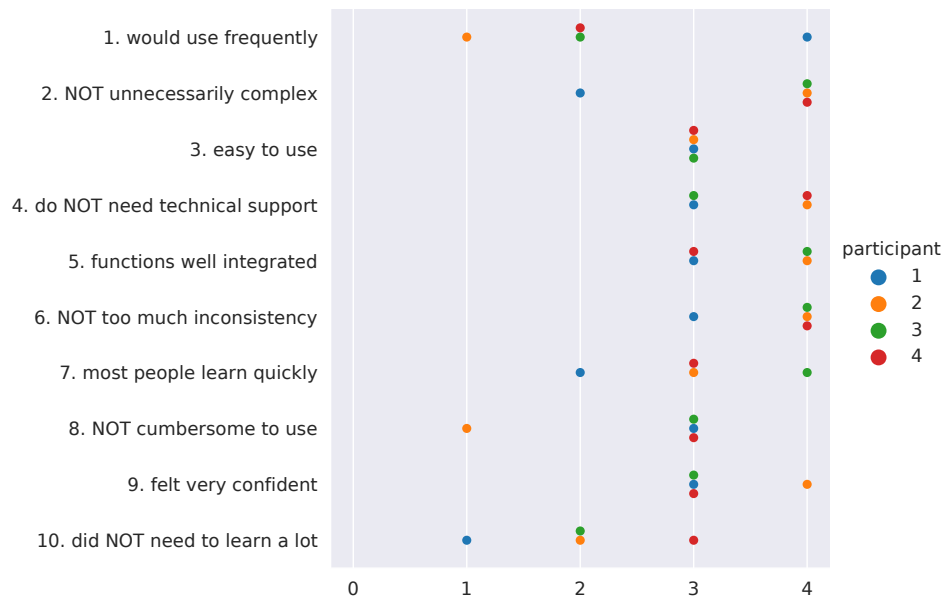

Fig. 9. Detailed answers to the System Usability Scale questionnaire. For better readability, every second question is inverted (see the additional NOT). All questions are normalized to a value between 0 (strongly disagree) and 4 (strongly agree) as in the score computation for the SUS [7].

all participants felt they were familiar with the system and ready to use it autonomously. They were then asked to solve a set of five predefined tasks with the Think Aloud method to comment on their intentions [30].

(1) Have movies with a budget of $\$ 250$ million or more made a profit? Which movie stands out?

(2) Do movies with a TMDb rating of 9 and above also have a good IMDb rating? Try to find reasons why these movies have such a good rating on TMDb.

(3) Look at movies that have a rating of 8 or higher on IMDb and TMDb and more than 1.5 million votes on IMDb. Does the budget correlate with the popularity of these movies? Which of these movies flopped in theaters?

(4) Which five movies from before 1950 are the most popular? Is their popularity also reflected in the number of votes on IMDb?

(5) Are movies that were released before 1939 and have an IMDb rating of 8 or more still popular today?

The experimenter took notes and recorded the participants' actions with their consent on video. Since we were interested in their individual feedback, the participants were encouraged to take their time with the tasks and to ask questions when needed. Overall, all five tasks took the experts between 20 and 40 minutes ( $M: 27 \mathrm{~min}, \sigma: 8 \mathrm{~min}$ ). After solving all of the given tasks, participants were asked to answer the System Usability Scale (SUS) questionnaire [7]. The expert review was concluded with a semi-structured interview on the user experience covering usability issues, features they liked, disliked, and missed, as well as their general experience of working with the LHRD. Based on the observations during the tasks, they were also asked about their individual style of interacting with the system. With the consent of our participants, the audio of the interviews was recorded for further analysis.

For analyzing the qualitative data (video recordings, notes, and interview recordings) we used an open coding approach to explore how the participants used our gesture set and to find usability issues. The relevant passages were then grouped by the interaction they relate to. Furthermore, we counted the number of times a participant used a specific gesture during the tasks of the review. 


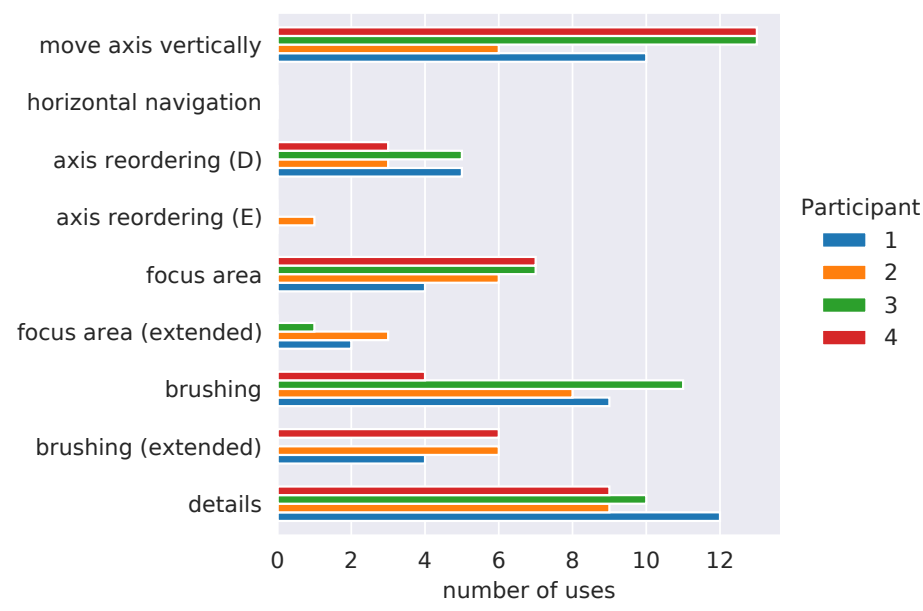

Fig. 10. The number of times each participant applied one of our proposed interactions during the tasks of the expert review.

\subsection{Results}

All participants were able to use our system and the multitouch gestures to successfully solve all of the given tasks with the dataset. They stated that the interaction vocabulary was suited to solve such tasks and comprehensively supported them in their visual analysis. However, P4 mentioned that "one needs a little time to get used to it" which is also reflected in statement 10 of the SUS questionnaire (see Figure 9). Overall, most of the user statements support the usability of our system which P1 described as "straight-forward" and "fun to use". P4 stated that "the gestures supported me in tackling the challenges of this big display".

Accessing Axes Vertically. Moving the axes vertically to bring regions of interest into reach was one of the most frequently used interactions (see Figure 10). It was typically used as a first step to bring a region of interest on an axis into reach or even just to access it more conveniently. P3 mentioned the vertical translation of axes as a feature they especially liked and commonly used to position regions of interest at eye level. In contrast, P2 rather crouched to access data close to the floor and stated in the interview that they preferred direct interaction whenever possible. Participants mainly preferred to drag the axis directly instead of using the step-wise variant with a fling which was only rarely used.

Navigating the Plot. Virtual navigation by scrolling through the axes with a horizontal fling was not used at all during the tasks. The participants rather navigated physically by walking to axes of interest. P2 explained that while scrolling is well-known, it entails lots of changes in the visual representation. Therefore, the experts avoided it in order to retain a mental map of axes positions which allowed them to locate and approach axes more quickly with physical navigation. In the interview, P1 stated that walking back and forth in order to switch between overview and details was "not disruptive". The other participants mentioned that they did not think of the virtual navigation because they did not need it.

Large Distance Axes Reordering. P2 mentioned during the exploration phase that they liked the idea of holding an axis and scrolling the other axes with a fling very much. However, during the tasks, 
this interaction was used only once. All participants preferred the long-distance axis dragging instead and P1, P3, and P4 mentioned the hyperbolic mapping of the horizontal axis dragging as a feature they especially liked. In the interview, P2 said it was an effective interaction yet felt "a little weird" due to the distance between touch gesture and manipulated target. In contrast, P4 suggested amplifying the non-linear mapping even more. P3 claimed that they preferred direct dragging in principle but had to acknowledge that the "nasty" feeling of prolonged touch on the fingertips would make that very uncomfortable in practice.

Focus Areas on Axes. Focus areas were used frequently during the evaluation tasks and P4 highlighted the importance and utility of focus areas for dense data. Task 3 and 5 required our participants to select movies from a certain date range and all of them created a focus area to inspect the date axis in more detail. Our participants also used a focus area in combination with a previous vertical translation to locally align the budget and revenue axes to a common scale for task 3 .

Both the brushing and the definition of focus areas involve defining a range on an axis. As a result, participants sometimes confused them. P1 and P2 suggested extra handles for one of them to resolve the ambiguity. P3 initially had problems creating a focus area because they expected to define the center point of the focus area with the hold gesture and hence proposed a pinch with four fingers as an alternative to distinguish them more clearly.

Data Selection, Filtering, and Details. Brushing was the only way to select a subset of data items and was therefore very frequently used to iteratively refine a selection over multiple attributes. In combination with the tap for showing details, the participants used it to read off exact values of a selection. $\mathrm{P} 4$ suggested in the interview to additionally display the values of a selection range during the interaction. Furthermore, they would have liked to show details for two attributes at once to be able to compare values directly.

Vertical Range Extension. The vertical range extensions of both the brushes and the focus areas allows users to fluently amplify their reach during interaction. The expert reviews show that the participants generally preferred to bring a region of interest into reach first by vertically dragging the axis and perform a brush directly afterward. However, when the participants realized a lack of range once they already started an interaction, they remembered and used the range extension technique (as it was intended). P1 stated in the interview that the range extension was "very helpful" but they often did not think of it straight away when planning their interactions ahead. While this shows that direct interaction is preferred, users need a reliable and seamless method to increase their interaction range.

Overall, our experts appreciated that the vertical range extensions and the large distance axes reordering unobtrusively extended their reach when required but generally preferred direct interaction when possible. The reviews also uncovered a lack of distinction between the gestures for brushing and creating a focus area. The fling-based horizontal scrolling was used only once and our participants instead favored physical navigation.

\subsection{Study Limitations}

Although we can draw first conclusions from the observations and the feedback given by our experts, our study only represents an initial evaluation primarily focused on finding general usability issues which should be improved in future iterations of our vocabulary. A subsequent user study with more participants is required to comprehensively and quantitatively evaluate the proposed gesture set as well as to compare it with other techniques such as distant interaction with mobile devices. The expert review was scoped to a predefined set of tasks which were designed to require the

Proc. ACM Hum.-Comput. Interact., Vol. 4, No. ISS, Article 199. Publication date: November 2020. 
experts to perform actions typical for PCPs such as selecting items in specific ranges, inspecting correlations, and spotting patterns across attributes. Thus, other tasks, a different dataset or a free exploration of the data could impact the behavior and interactions of participants. Similarly, the participants only used the system for a relative short time and the duration of an analysis might affect interaction patterns.

Interaction design is often affected by a bias towards interactions that are known from experience with prior interfaces and technologies [28]. While legacy-inspired interactions are easier to guess and learn, they are often not ideal for a task at hand. For gestural interaction, many users draw on their experience of popular applications such as Google Maps which established navigation through pinch, drag, and rotation gestures [41]. As a consequence, users tend to suggest and expect similar gestures. However, these gestures alone can often not cover the full range of required actions and therefore a larger gesture vocabulary is required. Once internalized such a vocabulary can be potentially more powerful, but before that the legacy bias can cause a more critical assessment.

Our current prototype was implemented to demonstrate and evaluate our proposed gesture set and therefore lacks some features a visual analysis system should provide. The experts suggested many features such as undo/redo, managing multiple selections, and configuration of colors and attributes that would improve the versatility and utility of our system.

\section{DISCUSSION AND LIMITATIONS}

Our prototype application of parallel coordinates on a wall-sized display addresses the issues of reachability and clutter with a gesture set that relies on short-range flings and bimanual gestures based on the concept of Hold-and-Move. While the expert reviews suggest that direct interaction is preferred whenever possible, the sheer size of the display makes interaction techniques necessary that virtually extend a user's reach beyond their physical capabilities. Our proposed gestures for extending reach effectively supported our experts in solving analysis tasks on such a display and were well received as they complement direct interaction by bringing regions of interest towards the user, fluently extending a brush or focus area during interaction, and boosting the drag of an axis up to the full width of the screen for axes reordering.

The focus areas successfully tackle the issue of clutter in parallel coordinates and can also be used to locally align axes to a common scale. However, the expert reviews revealed that our proposed hold and drag gesture for creating focus areas was too similar to the brushing and therefore confused our participants. As P3 suggested, this could be resolved by using bimanual pinch with two fingers or extra handles for either interaction as suggested by P1 and P2. Apart from the confusion of the range-based gestures, our experts stated that the gestures were comprehensible and easy to use. Manipulation based on the concept of Hold-and-Move was quickly embraced by our users to distinguish the target of the interaction.

Our fling-based cyclic horizontal scrolling of the PCP was designed to switch the focus to other axes while staying close to the display and thereby avoid the need for physical navigation. However, our participants only used this interaction once during the tasks of the expert reviews and instead walked along the screen as well as back and forth. As P2 emphasized, scrolling causes rapid visual changes that might be perceived as uncomfortable and overwhelming from up close. While physical navigation draws from embodied resources such as spatial memory and allows user to locate attributes more quickly, cyclic scrolling shifts all axes with a wrap-around and thereby invalidates the relative positions of axes to each other. Two participants stated that they did not need the virtual navigation and P1 mentioned that physical navigation was not "disruptive". This seems to reflect the findings of Ball et al. that physical navigation is mostly preferred over virtual navigation $[3,4]$. Yet, our study involved a short and focused analysis and the use of physical navigation 
could decrease in a longer session when fatigue can become an issue and in turn lead to a higher acceptance of virtual navigation.

In contrast to previous works, our multitouch gesture set facilitates interaction from up close and yet equips users with the means to reach the whole screen. However, the focus on touch as the only input modality is also a limitation of our work since users tend to step back to gain an overview of the whole visualization. Many forms of remote interaction with LHRDs have been presented in the related work-most prominently with mobile devices. Yet, holding a mobile device occupies one hand and is therefore incompatible with our bimanual gestures. As a consequence, we argue that mid-air gestures or speech input are well-suited to supplement our gesture set with the means of interaction from a distance. As Srinivasan et al. point out, multimodal interaction should "strive for synergy not equivalence" [46]. Similar to their work, a combination of different input modalities would facilitate direct interaction on the data such as brushing using multitouch whereas more complex or abstract actions would be provided by another modality.

Our successful adaptation and extension of the Hold-and-Move concept to PCPs on the large display encouraged us to think about how this concept could also be applied to smaller screens such as tabletops and vertical displays with a diagonal of 90 inches and below. In contrast to wall-sized displays the whole screen area is in reach for touch interaction, yet the screen size still offers sufficient space for two-handed interaction. Such screens could especially benefit from the distinction between background and foreground to manipulate different parts of the visualization as they are more densely drawn and multitouch gestures are often ambiguous.

The sheer size of LHRDs offers a huge potential for collaborative visual analysis. PCPs are well suited for collaboration as analysts can either work individually by focusing on separate axes in parallel, work closely together on an axis or a pair of axes, or support each other from varying distances to split up detail and overview between them. However, our system so far is focused on a single analyst and especially our navigation gestures conflict with loosely collaborating users as they globally shift the axes. Yet, our study questions the need for virtual navigation and shows that our participants preferred physical navigation which is better suited for collaborative scenarios. We believe that our system could already support some forms of close collaboration in which analysts interact in turns but this needs to be evaluated further. The integration of distant interaction techniques would strengthen collaboration as teams of analysts can perceive different levels of details at the same time and still interact with the visualization as shown by Langner et al. [23].

\section{CONCLUSION AND FUTURE WORK}

In this paper, we present a multitouch vocabulary for effective interaction with parallel coordinates visualized on large, high-resolution displays (LHRDs). Expert reviews confirmed that our gestures support users in visual analysis tasks on LHRDs by extending their reach while avoiding long-range swipes. However, the study also revealed that the gestures for range definition for brushing and focus areas can be easily confused. Furthermore, physical navigation was mostly preferred over cyclically scrolling the PCP for tasks of our study. Our participants preferred direct interaction when possible but appreciated our range extensions which allowed them to fluently extend a vertical range beyond their physical reach. They quickly embraced the principles of two-finger gestures for range specification and Hold-and-Move as the guiding principle for manipulating the arrangement of the PCP.

The participants of our expert reviews considered our gesture set as being comprehensive for typical PCP interactions but suggested typical system features to be implemented for a full data analysis system. In particular, undo and redo actions are needed. Currently, we only support explicitly resetting selections and focus areas but other actions such as navigation and axes switching are only reversible by performing the reverse interaction sequence. Furthermore, users need to be 
able to store different subsets of the data items during the filtering and analysis process and should be able to recall them for comparison later. In this regard, especially when considering the display size, inverse filtering would be a welcome addition.

Based on our results, future work should investigate how other standard visualization techniques could be adapted for multitouch interaction on LRHDs. The main challenge is to find an appropriate subset of gestures that is applicable globally across all visualizations, yet also to design gestures that are tailored to visualizations but do not interfere with the specific gestures of the other visualizations and also do not contradict the global gestures.

Our multitouch approach could work along with remote interaction through mid-air gestures or speech input. A multimodal interface could support interactions from a distance as well as from close up. Overall, we aim at integrating other complementary visualizations along with their corresponding remote and multitouch interaction techniques into a versatile coordinated multiview framework that is also able to take advantage of (almost) the entire screen for the individual visualizations. This would enable the user to choose the most suitable one among a variety of visualizations and to work at a personally favored distance or even take turns of using remote and close-up interaction.

\section{ACKNOWLEDGMENTS}

Our research has received funding from the German Aerospace Center (DLR) under Grant No. D/943/67270213. We thank the participants of our study for their time and feedback, the DLR Visual Analytics group Jena and the members of the Virtual Reality and Visualization Research Group at Bauhaus-Universität Weimar (http://www.uni-weimar.de/vr) for their support, and the reviewers of this paper for their constructive feedback.

\section{REFERENCES}

[1] Christopher Andrews, Alex Endert, and Chris North. 2010. Space to think: large high-resolution displays for sensemaking. In Proceedings of the 28th International Conference on Human Factors in Computing Systems, CHI 2010, Atlanta, Georgia, USA, April 10-15, 2010, Elizabeth D. Mynatt, Don Schoner, Geraldine Fitzpatrick, Scott E. Hudson, W. Keith Edwards, and Tom Rodden (Eds.). ACM, 55-64. https://doi.org/10.1145/1753326.1753336

[2] Christopher Andrews, Alex Endert, Beth Yost, and Chris North. 2011. Information visualization on large, highresolution displays: Issues, challenges, and opportunities. Information Visualization 10, 4 (2011), 341-355. https: //doi.org/10.1177/1473871611415997

[3] Robert Ball and Chris North. 2007. Realizing embodied interaction for visual analytics through large displays. Comput. Graph. 31, 3 (2007), 380-400. https://doi.org/10.1016/j.cag.2007.01.029

[4] Robert Ball, Chris North, and Doug A. Bowman. 2007. Move to improve: promoting physical navigation to increase user performance with large displays. In Proceedings of the 2007 Conference on Human Factors in Computing Systems, CHI 2007, San Jose, California, USA, April 28 - May 3, 2007, Mary Beth Rosson and David J. Gilmore (Eds.). ACM, 191-200. https://doi.org/10.1145/1240624.1240656

[5] Dominikus Baur, Bongshin Lee, and Sheelagh Carpendale. 2012. TouchWave: kinetic multi-touch manipulation for hierarchical stacked graphs. In Interactive Tabletops and Surfaces, ITS'12, Cambridge/Boston, MA, USA, November 11-14, 2012, Orit Shaer, Chia Shen, Meredith Ringel Morris, and Michael S. Horn (Eds.). ACM, 255-264. https: //doi.org/10.1145/2396636.2396675

[6] Anastasia Bezerianos and Petra Isenberg. 2012. Perception of Visual Variables on Tiled Wall-Sized Displays for Information Visualization Applications. IEEE Trans. Vis. Comput. Graph. 18, 12 (2012), 2516-2525. https://doi.org/10. 1109/TVCG.2012.251

[7] John Brooke. 1996. SUS-A quick and dirty usability scale. Usability evaluation in industry 189, 194 (1996), 4-7.

[8] Thorsten Büring, Jens Gerken, and Harald Reiterer. 2006. User Interaction with Scatterplots on Small Screens - A Comparative Evaluation of Geometric-Semantic Zoom and Fisheye Distortion. IEEE Trans. Vis. Comput. Graph. 12, 5 (2006), 829-836. https://doi.org/10.1109/TVCG.2006.187

[9] Mohammad Chegini, Shao Lin, Dirk Joachim Lehmann, Keith Andrews, and Tobias Schreck. 2017. Interaction Concepts for Collaborative Visual Analysis of Scatterplots on Large Vertically-Mounted High-Resolution Multi-Touch Displays. In Proceedings of the 10th Forum Media Technology and 3rd All Around Audio Symposium, St. Pölten, Austria, November 
29-30, 2017 (CEUR Workshop Proceedings, Vol. 2009), Wolfgang Aigner, Thomas Moser, Kerstin Blumenstein, Matthias Zeppelzauer, Michael Iber, and Grischa Schmiedl (Eds.). CEUR-WS.org, 90-96. http://ceur-ws.org/Vol-2009/fmtproceedings-2017-paper13.pdf

[10] Raimund Dachselt and Robert Buchholz. 2009. Natural throw and tilt interaction between mobile phones and distant displays. In Proceedings of the 27th International Conference on Human Factors in Computing Systems, CHI 2009, Extended Abstracts Volume, Boston, MA, USA, April 4-9, 2009, Dan R. Olsen Jr., Richard B. Arthur, Ken Hinckley, Meredith Ringel Morris, Scott E. Hudson, and Saul Greenberg (Eds.). ACM, 3253-3258. https://doi.org/10.1145/1520340.1520467

[11] Steven Mark Drucker, Danyel Fisher, Ramik Sadana, Jessica Herron, and m. c. schraefel. 2013. TouchViz: a case study comparing two interfaces for data analytics on tablets. In 2013 ACM SIGCHI Conference on Human Factors in Computing Systems, CHI '13, Paris, France, April 27 - May 2, 2013, Wendy E. Mackay, Stephen A. Brewster, and Susanne Bødker (Eds.). ACM, 2301-2310. https://doi.org/10.1145/2470654.2481318

[12] Niklas Elmqvist, Pierre Dragicevic, and Jean-Daniel Fekete. 2008. Rolling the Dice: Multidimensional Visual Exploration using Scatterplot Matrix Navigation. IEEE Trans. Vis. Comput. Graph. 14, 6 (2008), 1539-1148. https://doi.org/10.1109/ TVCG.2008.153

[13] G. W. Furnas. 1986. Generalized Fisheye Views. SIGCHI Bull. 17, 4 (April 1986), 16-23. https://doi.org/10.1145/22339 22342

[14] Jérémie Gilliot, Géry Casiez, and Nicolas Roussel. 2014. Direct and indirect multi-touch interaction on a wall display. In Proceedings of the 26th Conference on l'Interaction Homme-Machine, Villeneuve d'Ascq, France, October 28-31, 2014, Géry Casiez, Thomas Pietrzak, Olivier Chapuis, and Stéphane Conversy (Eds.). ACM, 147-152. https://doi.org/10. $1145 / 2670444.2670445$

[15] Faizan Haque, Mathieu Nancel, and Daniel Vogel. 2015. Myopoint: Pointing and Clicking Using Forearm Mounted Electromyography and Inertial Motion Sensors. In Proceedings of the 33rd Annual ACM Conference on Human Factors in Computing Systems, CHI 2015, Seoul, Republic of Korea, April 18-23, 2015, Bo Begole, Jinwoo Kim, Kori Inkpen, and Woontack Woo (Eds.). ACM, 3653-3656. https://doi.org/10.1145/2702123.2702133

[16] Mathias Heilig, Stephan Huber, Mischa Demarmels, and Harald Reiterer. 2010. ScatterTouch: a multi touch rubber sheet scatter plot visualization for co-located data exploration. In ACM International Conference on Interactive Tabletops and Surfaces, ITS 2010, Saarbrücken, Germany, November 7-10, 2010, Antonio Krüger, Johannes Schöning, Daniel Wigdor, and Michael Haller (Eds.). ACM, 263-264. https://doi.org/10.1145/1936652.1936707

[17] Julian Heinrich and Daniel Weiskopf. 2013. State of the Art of Parallel Coordinates. In Eurographics 2013 - State of the Art Reports, Girona, Spain, May 6-10, 2013, Mateu Sbert and László Szirmay-Kalos (Eds.). Eurographics Association, 95-116. https://doi.org/10.2312/conf/EG2013/stars/095-116

[18] Tom Horak, Sriram Karthik Badam, Niklas Elmqvist, and Raimund Dachselt. 2018. When David Meets Goliath: Combining Smartwatches with a Large Vertical Display for Visual Data Exploration. In Proceedings of the 2018 CHI Conference on Human Factors in Computing Systems, CHI 2018, Montreal, QC, Canada, April 21-26, 2018, Regan L. Mandryk, Mark Hancock, Mark Perry, and Anna L. Cox (Eds.). ACM, 19. https://doi.org/10.1145/3173574.3173593

[19] Mikkel R. Jakobsen and Kasper Hornbæk. 2015. Is Moving Improving?: Some Effects of Locomotion in Wall-Display Interaction. In Proceedings of the 33rd Annual ACM Conference on Human Factors in Computing Systems, CHI 2015, Seoul, Republic of Korea, April 18-23, 2015, Bo Begole, Jinwoo Kim, Kori Inkpen, and Woontack Woo (Eds.). ACM, 4169-4178. https://doi.org/10.1145/2702123.2702312

[20] Ulrike Kister, Konstantin Klamka, Christian Tominski, and Raimund Dachselt. 2017. GraSp: Combining Spatially-aware Mobile Devices and a Display Wall for Graph Visualization and Interaction. Comput. Graph. Forum 36, 3 (2017), 503-514. https://doi.org/10.1111/cgf.13206

[21] Robert Kosara. 2011. Indirect multi-touch interaction for brushing in parallel coordinates. In Visualization and Data Analysis 2011, San Francisco Airport, CA, USA, fanuary 24-25, 2011 (SPIE Proceedings, Vol. 7868), Pak Chung Wong, Jinah Park, Ming C. Hao, Chaomei Chen, Katy Börner, David L. Kao, and Jonathan C. Roberts (Eds.). SPIE, 786809. https://doi.org/10.1117/12.872645

[22] Alexander Kulik, Jan Dittrich, and Bernd Froehlich. 2012. The hold-and-move gesture for multi-touch interfaces. In Mobile HCI '12, Proceedings of the 14th international conference on Human-computer interaction with mobile devices and services, San Francsico, CA, USA, September 21-24, 2012, Elizabeth F. Churchill, Sriram Subramanian, Patrick Baudisch, and Kenton O’Hara (Eds.). ACM, 49-58. https://doi.org/10.1145/2371574.2371583

[23] Ricardo Langner, Ulrike Kister, and Raimund Dachselt. 2019. Multiple Coordinated Views at Large Displays for Multiple Users: Empirical Findings on User Behavior, Movements, and Distances. IEEE Trans. Vis. Comput. Graph. 25, 1 (2019), 608-618. https://doi.org/10.1109/TVCG.2018.2865235

[24] Bongshin Lee, Petra Isenberg, Nathalie Henry Riche, and Sheelagh Carpendale. 2012. Beyond Mouse and Keyboard: Expanding Design Considerations for Information Visualization Interactions. IEEE Trans. Vis. Comput. Graph. 18, 12 (2012), 2689-2698. https://doi.org/10.1109/TVCG.2012.204 
[25] Lars Lischke, Sven Mayer, Jan Hoffmann, Philipp Kratzer, Stephan Roth, Katrin Wolf, and Pawel Wozniak. 2017. Interaction techniques for window management on large high-resolution displays. In Proceedings of the 16th International Conference on Mobile and Ubiquitous Multimedia, MUM 2017, Stuttgart, Germany, November 26 - 29 , 2017, Niels Henze, Pawel Wozniak, Kaisa Väänänen, Julie Williamson, and Stefan Schneegass (Eds.). ACM, 241-247. https://doi.org/10.1145/3152832.3152852

[26] Can Liu, Olivier Chapuis, Michel Beaudouin-Lafon, Eric Lecolinet, and Wendy E. Mackay. 2014. Effects of display size and navigation type on a classification task. In CHI Conference on Human Factors in Computing Systems, CHI'14, Toronto, ON, Canada - April 26 - May 01, 2014, Matt Jones, Philippe A. Palanque, Albrecht Schmidt, and Tovi Grossman (Eds.). ACM, 4147-4156. https://doi.org/10.1145/2556288.2557020

[27] Mingyu Liu, Mathieu Nancel, and Daniel Vogel. 2015. Gunslinger: Subtle Arms-down Mid-air Interaction. In Proceedings of the 28th Annual ACM Symposium on User Interface Software \& Technology, UIST 2015, Charlotte, NC, USA, November 8-11, 2015, Celine Latulipe, Bjoern Hartmann, and Tovi Grossman (Eds.). ACM, 63-71. https://doi.org/10.1145/2807442. 2807489

[28] Meredith Ringel Morris, Andreea Danielescu, Steven Mark Drucker, Danyel Fisher, Bongshin Lee, m. c. schraefel, and Jacob O. Wobbrock. 2014. Reducing legacy bias in gesture elicitation studies. Interactions 21, 3 (2014), 40-45. https://doi.org/10.1145/2591689

[29] Mathieu Nancel, Julie Wagner, Emmanuel Pietriga, Olivier Chapuis, and Wendy E. Mackay. 2011. Mid-air pan-and-zoom on wall-sized displays. In Proceedings of the International Conference on Human Factors in Computing Systems, CHI 2011, Vancouver, BC, Canada, May 7-12, 2011, Desney S. Tan, Saleema Amershi, Bo Begole, Wendy A. Kellogg, and Manas Tungare (Eds.). ACM, 177-186. https://doi.org/10.1145/1978942.1978969

[30] Jakob Nielsen. 1993. Usability engineering. Academic Press.

[31] PQLabs. [n.d.]. Support Center. https://www.pqlabs.com/support_wall.html. Accessed: 03/2019.

[32] Arnaud Prouzeau, Anastasia Bezerianos, and Olivier Chapuis. 2017. Evaluating Multi-User Selection for Exploring Graph Topology on Wall-Displays. IEEE Trans. Vis. Comput. Graph. 23, 8 (2017), 1936-1951. https://doi.org/10.1109/ TVCG.2016.2592906

[33] Renata G. Raidou, Martin Eisemann, Marcel Breeuwer, Elmar Eisemann, and Anna Vilanova. 2016. OrientationEnhanced Parallel Coordinate Plots. IEEE Trans. Vis. Comput. Graph. 22, 1 (2016), 589-598. https://doi.org/10.1109/ TVCG.2015.2467872

[34] Fateme Rajabiyazdi, Jagoda Walny, Carrie Mah, John Brosz, and Sheelagh Carpendale. 2015. Understanding Researchers' Use of a Large, High-Resolution Display Across Disciplines. In Proceedings of the 2015 International Conference on Interactive Tabletops \& Surfaces, ITS 2015, Funchal, Portugal, November 15-18, 2015, Nuno Jardim Nunes, Enrico Costanza, Patrick Olivier, and Johannes Schöning (Eds.). ACM, 107-116. https://doi.org/10.1145/2817721.2817735

[35] Khairi Reda, Andrew E. Johnson, Michael E. Papka, and Jason Leigh. 2015. Effects of Display Size and Resolution on User Behavior and Insight Acquisition in Visual Exploration. In Proceedings of the 33rd Annual ACM Conference on Human Factors in Computing Systems, CHI 2015, Seoul, Republic of Korea, April 18-23, 2015, Bo Begole, Jinwoo Kim, Kori Inkpen, and Woontack Woo (Eds.). ACM, 2759-2768. https://doi.org/10.1145/2702123.2702406

[36] Patrick Riehmann, Gabriela Molina León, Joshua Reibert, Florian Echtler, and Bernd Froehlich. 2020. Short-Contact Touch-Manipulation of Scatterplot Matrices on Wall Displays. Computer Graphics Forum (2020). https://doi.org/10. 1111/cgf.13979

[37] Patrick Riehmann, Joshua Reibert, Jens Opolka, and Bernd Fröhlich. 2018. Touch the Time: Touch-Centered Interaction Paradigms for Time-Oriented Data. In Eurographics Conference on Visualization, EuroVis 2018, Short Papers, Brno, Czech Republic, 4-8 June 2018, Jimmy Johansson, Filip Sadlo, and Tobias Schreck (Eds.). Eurographics Association, 113-117. https://doi.org/10.2312/eurovisshort.20181088

[38] Jonathan C. Roberts, Panagiotis D. Ritsos, Sriram Karthik Badam, Dominique Brodbeck, Jessie Kennedy, and Niklas Elmqvist. 2014. Visualization beyond the Desktop-the Next Big Thing. IEEE Computer Graphics and Applications 34, 6 (2014), 26-34. https://doi.org/10.1109/MCG.2014.82

[39] Richard C. Roberts, Robert S. Laramee, Gary A. Smith, Paul Brookes, and Tony D’Cruze. 2019. Smart Brushing for Parallel Coordinates. IEEE Trans. Vis. Comput. Graph. 25, 3 (2019), 1575-1590. https://doi.org/10.1109/TVCG.2018.2808969

[40] George G. Robertson, Mary Czerwinski, Patrick Baudisch, Brian Meyers, Daniel C. Robbins, Greg Smith, and Desney S. Tan. 2005. The Large-Display User Experience. IEEE Computer Graphics and Applications 25, 4 (2005), 44-51. https: //doi.org/10.1109/MCG.2005.88

[41] Vít Rusnák, Caroline Appert, Olivier Chapuis, and Emmanuel Pietriga. 2018. Designing Coherent Gesture Sets for Multi-scale Navigation on Tabletops. In Proceedings of the 2018 CHI Conference on Human Factors in Computing Systems, CHI 2018, Montreal, QC, Canada, April 21-26, 2018, Regan L. Mandryk, Mark Hancock, Mark Perry, and Anna L. Cox (Eds.). ACM, 142. https://doi.org/10.1145/3173574.3173716

[42] Jeffrey M. Rzeszotarski and Aniket Kittur. 2014. Kinetica: naturalistic multi-touch data visualization. In CHI Conference on Human Factors in Computing Systems, CHI'14, Toronto, ON, Canada - April 26 - May 01, 2014, Matt Jones, Philippe A 
Palanque, Albrecht Schmidt, and Tovi Grossman (Eds.). ACM, 897-906. https://doi.org/10.1145/2556288.2557231

[43] Ramik Sadana, Meeshu Agnihotri, and John T. Stasko. 2018. Touching Data: A Discoverability-based Evaluation of a Visualization Interface for Tablet Computers. CoRR abs/1806.06084 (2018). arXiv:1806.06084 http://arxiv.org/abs/1806. 06084

[44] Ramik Sadana and John T. Stasko. 2014. Designing and implementing an interactive scatterplot visualization for a tablet computer. In International Working Conference on Advanced Visual Interfaces, AVI 2014, Como, Italy, May 27-29, 2014, Paolo Paolini and Franca Garzotto (Eds.). ACM, 265-272. https://doi.org/10.1145/2598153.2598163

[45] Ramik Sadana and John T. Stasko. 2016. Designing Multiple Coordinated Visualizations for Tablets. Comput. Graph Forum 35, 3 (2016), 261-270. https://doi.org/10.1111/cgf.12902

[46] Arjun Srinivasan, Bongshin Lee, Nathalie Henry Riche, Steven M. Drucker, and Ken Hinckley. 2020. InChorus: Designing Consistent Multimodal Interactions for Data Visualization on Tablet Devices. In CHI '20: CHI Conference on Human Factors in Computing Systems, Honolulu, HI, USA, April 25-30, 2020, Regina Bernhaupt, Florian 'Floyd' Mueller, David Verweij, Josh Andres, Joanna McGrenere, Andy Cockburn, Ignacio Avellino, Alix Goguey, Pernille Bjøn, Shengdong Zhao, Briane Paul Samson, and Rafal Kocielnik (Eds.). ACM, 1-13. https://doi.org/10.1145/3313831.3376782

[47] Theophanis Tsandilas, Anastasia Bezerianos, and Thibaut Jacob. 2015. SketchSliders: Sketching Widgets for Visual Exploration on Wall Displays. In Proceedings of the 33rd Annual ACM Conference on Human Factors in Computing Systems, CHI 2015, Seoul, Republic of Korea, April 18-23, 2015, Bo Begole, Jinwoo Kim, Kori Inkpen, and Woontack Woo (Eds.). ACM, 3255-3264. https://doi.org/10.1145/2702123.2702129

[48] Ulrich von Zadow, Wolfgang Büschel, Ricardo Langner, and Raimund Dachselt. 2014. SleeD: Using a Sleeve Display to Interact with Touch-sensitive Display Walls. In Proceedings of the Ninth ACM International Conference on Interactive Tabletops and Surfaces, ITS 2014, Dresden, Germany, November 16 - 19, 2014, Raimund Dachselt, T. C. Nicholas Graham, Kasper Hornbæk, and Miguel A. Nacenta (Eds.). ACM, 129-138. https://doi.org/10.1145/2669485.2669507

[49] Bowen Yu and Xiaoru Yuan. 2011. Parallel Coordinates on iPad. In Poster Proceedings of IEEE Pacific Visualization Symposium (PacificVis 2011). IEEE, 21-22.

Received July 2020; revised August 2020; accepted September 2020 